\title{
CdSe Quantum dots Molecular Beacon Hybridization with Target Telomerase DNA Specificity

\author{
Linyan Shen ${ }^{\mathrm{a}}$, Ming Ying ${ }^{* \mathrm{~b}}$, Pengfei Deng ${ }^{\mathrm{c}}$,
}

\author{
School of Chemistry and Chemical Engineering, Tianjin University of Technology, Tianjin 300384, \\ China
}

a315447503@qq.com, bym8312@qq.com,,1030250850@qq.com

\begin{abstract}
Key Words: CdSe Quantum Dots, Molecular Beacon, Telomerase DNA, one-pot approach Abstract: Quantum Dots are powerful imaging and tracking tools for a wide range of biomedical applications, especially in preparing molecular beacons, but its use is limited by the sensitivity of the electrostatic binding between streptavidin and QDs. In this investigation, we used EDC as a crosslinker and NHS as a stabilizer to activate the carboxyl groups of carboxyl-modified CdSe QDs for direct reaction with primary amines of amino-modified single-stranded DNA. The results suggest that CdSe QDs can be applied for preparing the molecular beacon hybridized with specific telomerase DNA.
\end{abstract}

\section{Introduction}

Quantum dots ( QDs ) are a kind of nanoparticles, which can be zapped with laser light and generate fluorescence. Compared with other organic dyes, it has so many advantages that have already been developed into fluorescent bioprobe[1-3]. Among them, the emission spectroscopy of CdSe and $\mathrm{ZnS}$ could cover the whole visible area[4] and has excellent properties, such as rapid non-liner optical response[5-6] and luminescence at the room temperature[7-8]. Therefore, QDs are powerful imaging and tracking tools for a wide range of biomedical applications such as disease diagnoses[9] and prognoses[10], tracking cell/protein interactions[11] and cell vitality[12].

However, what could be observed after QD conjugation is a dramatic decrease of the quantum yield in water, in addition, the number of ssDNA strands conjugated to each QD is difficult to control. Streptavidin as a kind of positively charged protein could be absorbed electrostatically onto QDs followed conjugation with biotin-functionalized DNA with a negatively charged surface. However, the sensitivity of the electrostatic binding between streptavidin and QDs to ionic strengths limits the use of this approach. During the process of conjugation, QDs often aggregate at high ionic strength, which leads to a low yield of QD-DNA conjugates. Further more, due to the relatively large size of streptavidin molecules, which occupy the surface of QDs, the number of conjugated ssDNAstrands is usually small.

Therefore, in this study we used EDC and NHS as crosslinker and stabilizer respectively to activate the carboxyl groups of carboxyl-modified CdSe QDs for direct reaction with primary amines of amino-modified single-stranded DNA. The experimental procedure is simple with shorter reaction time and higher yield.

\section{Preparation of Molecular Beacon}

Firstly, the CdSe quantum dots $(50 \mu \mathrm{L}, 5 \mu \mathrm{M}$, prepared in our lab before) were diluted with TE buffer to $250 \mathrm{ml}$, and then EDC $(5 \mu \mathrm{L}, 50 \mathrm{mM})$ and Sulfo-NHS $(5 \mu \mathrm{L}, 50 \mathrm{mM})$ were added in, as the connection agent, to react for $15 \mathrm{~min}$ by oscillating at room temperature. Secondly, The 3 ' end of Dabcyl modified, 5' end amino modified DNA was added into the reaction for another 2 hours oscillating. Finally, after the reaction, the above-mentioned solution should be diluted with TE buffer to $500 \mathrm{ml}$, and using ultrafiltration centrifugal tube $(10 \mathrm{KD})$ for centrifugation $(6000 \mathrm{rpm}$, $25 \mathrm{~min})$. Repeat the procedures for three times to obtain the purified molecular beacon. The reaction 
process of molecular beacon is showed in Fig.1.

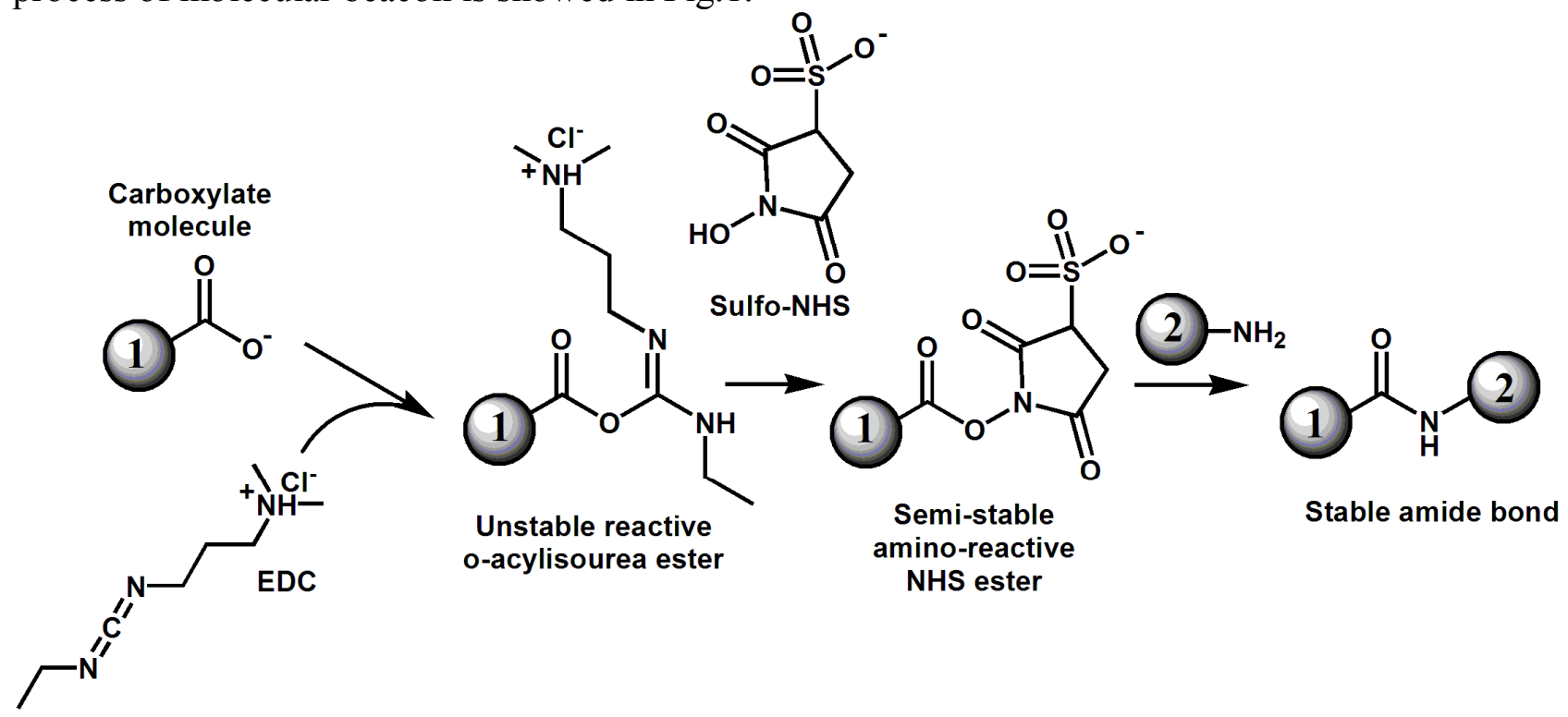

Fig.1Sulfo-NHS plus EDC crosslinking reaction scheme

\section{DNA and Chemicals}

DNA oligonucleotides were purchased from TaKaRa (Dalian, China). DNA oligonucleotides were dissolved in TE buffer $(100 \mathrm{mM} / \mathrm{L}$ Tris $(\mathrm{pH} 7.4)+10 \mathrm{mM} / \mathrm{L}$ EDTA $(\mathrm{pH}$ 8.0)) with desired concentrations before conjugation. Sequences for the DNA strands and modifications used are shown in Table 1.

Table1 DNA Sequence and Modifications Used in This Work

\begin{tabular}{cr}
\hline Name & Sequences and modifications (listed from 5' to 3') \\
\hline Amino, Dabcyl-modified DNA & 5'-NH 2 -C6-GTGACCGTGGTTTCTGTGTGGTGTCAC-3'-Da \\
bcyl \\
Target-DNA & 5'-GTGACACCACACAGAAACCACGGTCAC-3'. \\
Cy3, Dabcyl-modified DNA & 5'-Cy3-GTGACCGTGGTTTCTGTGTGGTGTCAC-3'-Dabcyl. \\
\hline
\end{tabular}

*Dabcyl: Dark Quencher 400-550 nm

$*$ Cy3: Aryl $=\mathrm{N}^{+}=\mathrm{CH}[\mathrm{CH}=\mathrm{CH}]_{2}-\mathrm{N}=$ Aryl

Cy3 dye ( $\sim 550 \mathrm{~nm}$ excitation, $\sim 570 \mathrm{~nm}$ emissions)can be excited using the $532 \mathrm{~nm}$ laser line, is a traditional orange-fluorescent label for protein and nucleic acid conjugates used for imaging, flow cytometry, and genomic applications.

N-Ethyl-N'-(3-dimethylaminopropyl) carbodiimide hydrochloride (EDC) and Sulfo-N-hydroxysulfosuccinimide sodium salt (Sulfo-NHS) were from HEOWNS (Tianjin). EDC reacts with carboxylic acid groups to form an active O-acylisourea intermediate, which is easily displaced by primary amino groups via nucleophilic attack in the reaction mixture. Then the primary amine forms an amide bond with the original carboxyl group, resulting a releasing EDC by-product as a soluble urea derivative. Sulfo-NHS is often included in EDC coupling protocols to improve efficiency or create dry-stable intermediatesdue to the Sulfo-NHS ester is more stable than the $\mathrm{O}$-acylisourea intermediate while allowing for efficient conjugation to primary amines at physiologic $\mathrm{pH}$. 


\section{Analysis method}

UV-Vis absorption and fluorescence (FL) spectra were measured at room temperature with a Hitachi U-3310 spectrophotometer and a Hitachi F-4500 fluorescence spectrophotometer (Japan) respectively. All the colloid solutions of CdSe QDs samples and CdSe molecular beacon samples were diluted 3-time with deionization water. FL spectra were taken at the excitation wavelength $\lambda$ ex $=380 \mathrm{~nm}$ with a $150 \mathrm{w}$ xenon lampas light source. Slit width of emission channelswas set to $10 \mathrm{~nm}$ and measuring wavelength range was $400 \mathrm{~nm}$ to $700 \mathrm{~nm}$.

\section{Results and discussion}

Conjugation of Carboxyl-QDs with Amino-DNA: To make sure that we obtained the conjugation of Carboxyl-QDs with Amino-DNA shown in Fig.2, we examined the fluorescence of CdSe QDs and the conjugation respectively. The results shown in Fig.3 displayed that the fluorescence intensity had a drastically decline from 2250 to 810 at $550 \mathrm{~nm}$, which was due to the mechanism of fluorescence resonance energy transfer (FRET). When the QDs (fluorophore) was assembled to the modified DNA, having a quencher, the excitation spectra of the quencher and the fluorophore overlapped, the energy transferred from the fluorophore (donor) to the quencher (acceptor), resulting the fluorescence intensity of QDs decreased.

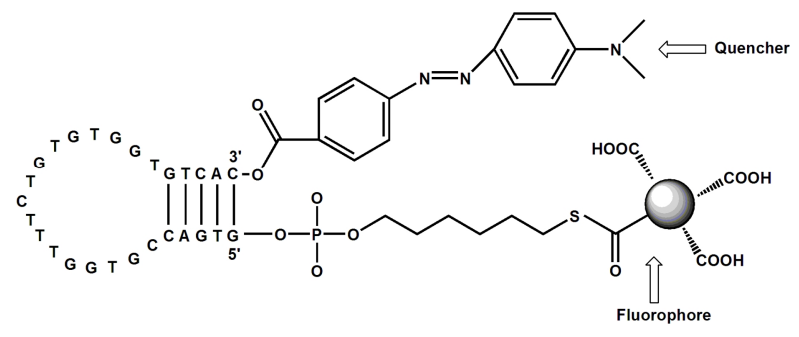

Fig. 2 The Structure of Molecular Beacon

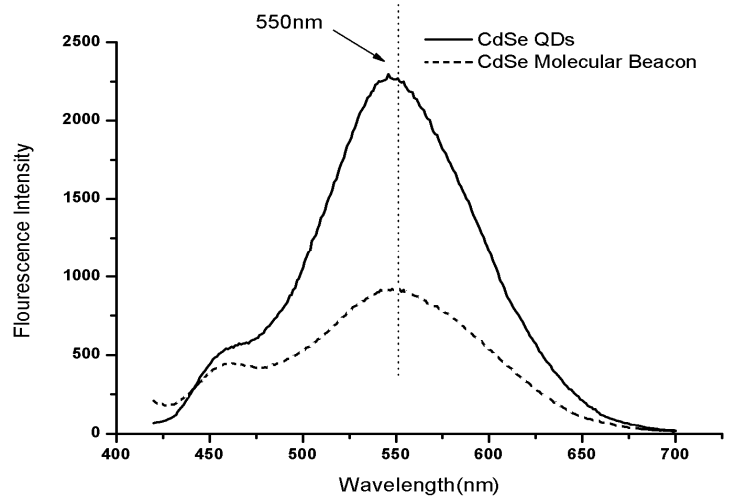

Fig.3 The Fluorescence of CdSe QDs and the Conjugation

Target-DNA Assay : The target chain (nucleic acid:molecular beacon=5:1) as added into the solution of molecular beacon, and then diluted with TE buffer ( $\mathrm{pH} 7.6)$ to $300 \mu \mathrm{L}$. The samples were loaded in a 96 -well PCR plate and sealed with a heated $\left(105^{\circ} \mathrm{C}\right)$ plastic cover to avoid evaporation. The samples were analyzed using a PCR thermocycler (B960, ADVANCE). The cyclic DNA was open by heating; then, the instrument was first programmed to anneal the samples to $55^{\circ} \mathrm{C}$, heat up to $75^{\circ} \mathrm{C}$ and hold for $5 \mathrm{~min}$; last, the samples would be conserved at $4^{\circ} \mathrm{C}$ for 2 hours before taking out. After reaction, fluorescence was measured again shown Fig.4, the result described that the originally quenched fluorescence partially restored. The same procedures were performed using the Cy3 Molecular Beacon as a control group shown Fig.5. 


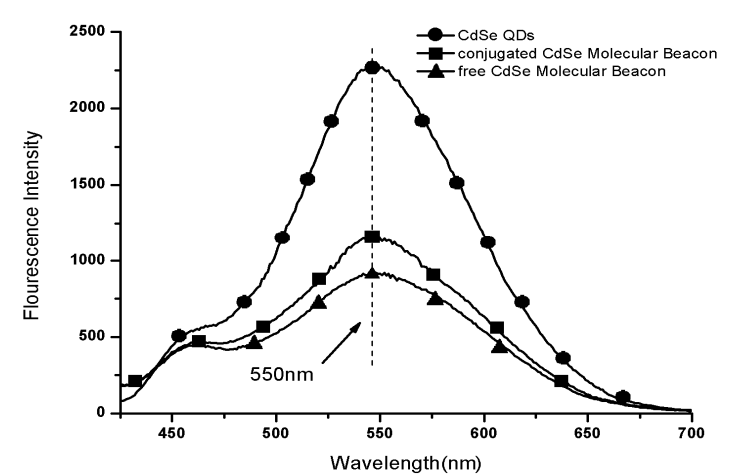

Fig.4 The Variational Fluorescence Curve of CdSe Molecular Beacon

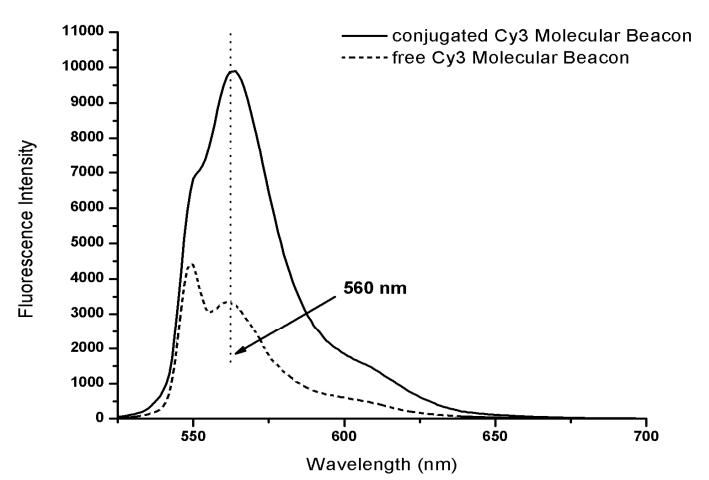

Fig. 5 The Variational Fluorescence Curve of Cy3 Molecular Beacon Binding to The Target Chain

\section{Conclusions}

In summary, we synthetized Molecular Beacon using the carboxyl-modified CdSe QDs and the amino-modified single-stranded DNA via the peptide bond. We measured the fluorescence intensity of the molecular beacon and the conjugated $\mathrm{Cy} 3$ molecular beacon respectively, the results showed that the FRET phenomenon disappeared and the fluorescence intensity recovered to the former level. Therefore, the conjugated $\mathrm{Cy} 3$ molecular beacon could be better used in biological experiments.

\section{Acknowledgements}

This research received funding from Science and technology correspondent project, which is supported by Tianjin Municipal Science and Technology Commission (Grant No.14JCTPJC00479).

\section{References}

[1] I.L. Medintz, H.T. Uyeda, E.R. Goldman, H. Mattoussi. Nature Mat.,Vol. 4 (2005), p. 435

[2] C. Jianrong, M. Yuqing, H. Nongyue, W. Xiaohua, L. Sijiao. Biotechnol. Adv., Vol. 22 (2004), p. 505

[3] F. Wang, B.W. Tan, Y. Zhang, X. Fan, M. Wang. Nanotechnol,Vol. 17(2006), p. 1

[4] HuaibinShen, Hongzhe Wang, Zhaojun Tang, Jin ZhongNiu, Shiyun Lou, Zuliang Du and Lin Song Li. Cryst. Eng. Comm., Vol. 11 (2009), p.1733

[5] M.A. Hines, P. Guyot-Sionnest. J. Phys. Chem., 1996, 100: 468

[6] C.A. Leatherdale, W.K. Woo, F.V. Mikulec and M.G. Bawendi. J. Phys. Chem. B, Vol. (106) 2002, p. 7619

[7] Xiaobo Chen, Yongbing Lou, Anna C. Samia and Clemens Burda. Nano Lett., Vol. 3 (2003), p. 799

[8] Christian Kirchner, Tim Liedl, Stefan Kudera, Teresa Pellegrino, Almudena Muñoz Javier, Hermann E. Gaub, Sonja Stölzle, N. Fertig and Wolfgang J. Parak. Nano Lett., Vol. 5 (2005), p. 331

[9] J.M. Klostranec, W.C.W. Chan. Adv. Mat.,Vol. 18 (2006), p. 1953

[10]C. Chen, H.S. Xia, Y.P. Gong, J. Peng, C.W. Peng, M.B. Hu. Biomaterials, Vol. 31 (2010), p. 8818

[11]S.J. Rosenthal, J.C. Chang, O. Kovtun, J.R. McBride. Chemistry \&Biology, Vol. 18 (2011), p. 10

[12]A.M. Derfus, S.N. Bhatia. Nano let.,Vol. 4(2004), p. 11 